\title{
Mass Consistency
}

National Cancer Institute

\section{Source}

National Cancer Institute. Mass Consistency. NCI Thesaurus. Code C90420.

A description about the density of a mass. 